\title{
Nonlinear Pulse Shaping in Optical Fibres with a Neural Network
}

\author{
Sonia Boscolo ${ }^{\mathbf{1}}$ and Christophe Finot ${ }^{2}$ \\ ${ }^{1}$ Aston Institute of Photonic Technologies, Aston University, Birmingham B4 7ET, United Kingdom \\ ${ }^{2}$ Laboratoire Interdisciplinaire Carnot de Bourgogne, Université de Bourgogne Franche-Comté, Dijon, France \\ s.a.boscolo@aston.ac.uk
}

\begin{abstract}
We use a machine-learning based paradigm to solve the direct and inverse problems relating to the shaping of optical pulses that occurs upon nonlinear propagation in optical fibres with a neural network. (C) 2020 The Author(s)
\end{abstract}

\section{Introduction}

The past decade has seen significant impact of machine learning on basic research, with the use of advanced algorithms tools in data analysis resulting in new insights into many areas of science. In the field of photonics, machine learning methods have been applied in various ways to optimise and analyse the output of an optical fibre system - see, e.g., [1-4]. Pulse shaping based on nonlinear phenomena in optical fibres has become a remarkable tool to tailor the spectral and temporal content of light signals [5], leading to the generation of a large variety of optical waveforms such as ultra-short compressed pulses, parabolic-, triangular- and rectangular-profiled pulses. Yet, due to the typically wide range of degrees of freedom involved, predicting the behaviour of nonlinear pulse shaping can be computationally demanding, especially when dealing with inverse problems. This potentially creates a severe bottleneck in using numerical techniques to design and optimise experiments in real time.

Here, we present a solution to this problem using machine learning to solve both the direct and inverse problems relating to pulse shaping with a neural network (NN), bypassing the need for numerical solution of the governing propagation model. Specifically, we show how the network accurately predicts the temporal and spectral intensity profiles of the pulses that form upon nonlinear propagation in fibres with both normal and anomalous dispersion. We also demonstrate the ability of the $\mathrm{NN}$ to determine the nonlinear propagation properties from the pulses observed at the fibre output and to classify the output pulses according to the initial pulse shape. Furthermore, we show how our model can handle the nonlinear shaping of initially chirped pulses.

\section{Model-free direct and inverse problems}

The data from numerical simulations of the nonlinear Schrödinger equation (NLSE) is used to train a NN and validate its predictions. We employ a feed-forward $\mathrm{NN}$ relying on the Bayesian regularisation back propagation algorithm and including three hidden layers with fourteen neurons each. The NN learns the NLSE model from an ensemble of several thousand simulation data (both temporal and spectral) corresponding to a mix of initial pulse shapes and randomly chosen combinations of input parameters: normalised propagation length $\xi$ and soliton-order number $N$. After training, the $\mathrm{NN}$ is tested on a distinct ensemble of data that is not used in the training phase.

Figure 1(a-b) shows examples of the temporal and spectral profiles of an initially Gaussian pulse obtained from the network for selected input parameters. The predictions from the algorithm show excellent agreement with the results of the NLSE propagation model over most part of the pulse shape and spectrum. The network is able to reproduce the large temporal and spectral broadening experienced by the pulse upon propagation in the normal dispersion regime. With anamorphic sampling of its output, the network is also able to resolve the details of the temporally compressed pulse and the concomitant more complex structure of the spectrum that are observed after propagation at anomalous dispersion. Nevertheless, some discrepancies with the expected results are visible, and are more pronounced in the anomalous regime in which the propagation dynamics are more complex and involve compression and/or splitting stages over short propagation distances. The distributions of values of the (normalised) mean squared deviations of the NN predictions from the NLSE simulation results for the output pulse shape and spectrum over the sample space (not shown here) indicate that, remarkably, more than $90 \%$ of the error realisations are well confined to values below 0.02 , but some error values are spread out over a wider range. This deviation mostly occurs in the anomalous dispersion regime.

For the inverse problem at hand, we ask the trained network to categorise new unlabelled simulated output pulses according to the initial waveform and to retrieve the associated propagation parameters $\xi$ and $N$ as well as the dispersion regime of the fibre. The results are summarised in Fig. 1(c-d). The estimation errors on the propagation length [Fig. 1(c)] and soliton-order number (defined as the difference between the predicted parameter value and the target value extracted from the NLSE simulation data) are close to zero for all test realisations except those corresponding to short propagation lengths or low input powers, for which the changes in the temporal and 
spectral shapes of the propagating pulse are negligibly small, thus leading to similar shapes for different $\xi$ or $N$. After exclusion of the data points falling into the critical parameter region, we can expect errors on $\xi$ and $N$ with root mean squared deviations of 0.085 and 0.076 , respectively [Fig. 1(d)]. The network is able to work out the sign of the fibre dispersion perfectly. The classification accuracy of the NN algorithm is remarkably high, with a number of classification errors representing less than $0.01 \%$ of the total number of input samples. Initial Gaussian, hyperbolic secant and parabolic pulses are detected correctly by the model. Moreover, the errors in the identification of super-Gaussian pulses occur in regions of the parameter space where we know that the NN algorithm has difficulty making correct predictions, that is, for short propagation lengths and in the anomalous dispersion regime of the fibre.
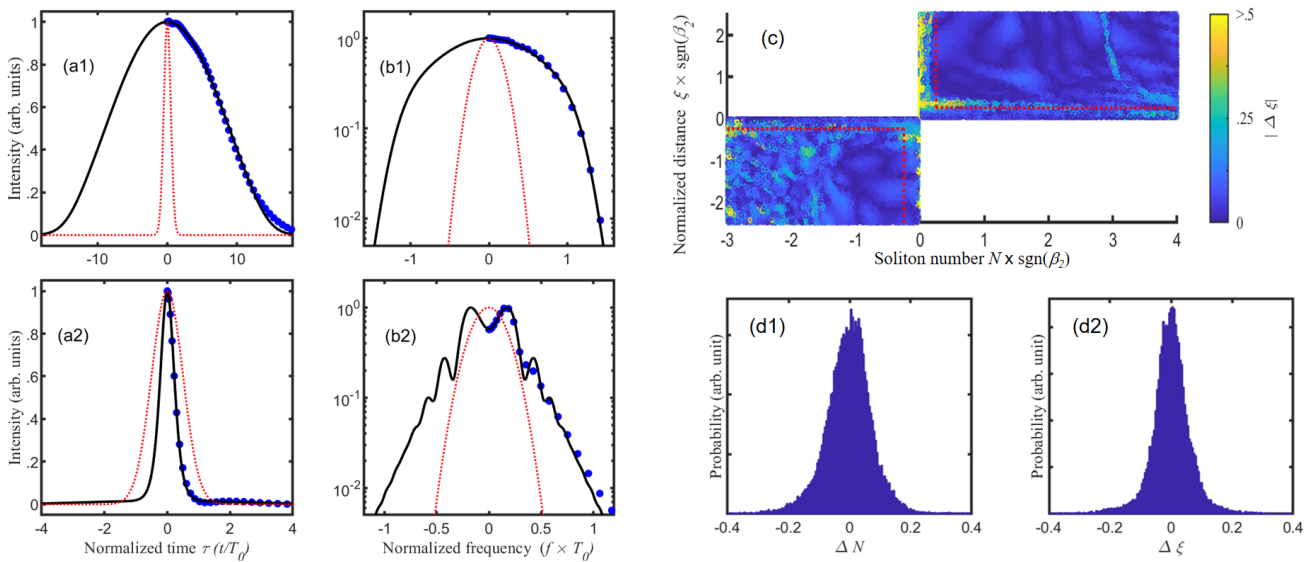

Fig. 1. (a-b) Direct shaping problem. Temporal (panels 1) and spectral (panels 2) intensity profiles of an initial Gaussian pulse in (a) a normally dispersive fibre with $\xi=2$ and $N=4$, and (b) an anomalously dispersive fibre with $\xi=1.8$ and $N=2.5$. The predictions from the $\mathrm{NN}$ (blue circles) are compared with the results of NLSE numerical simulations (black curves). Also shown are the input intensity profiles (red dotted curves). (c-d) Inverse shaping problem. (c) Map of estimation error values on the normalised propagation length $\xi$ in the two-dimensional space $(N, \xi)$ for both normal and anomalous dispersion, when the NN is interrogated with randomly chosen new simulated output pulses from the fibre corresponding to an unlabelled mix of input Gaussian, hyperbolic secant, parabolic and super-Gaussian pulses. The red dashed lines delimit the data domain that is used for the statistical error analysis. (d) Distribution densities of the estimation errors on $N$ (subplot d1) and $\xi$ (subplot $\mathrm{d} 2$ ).

We also investigate the nonlinear shaping of initially chirped pulses, for which the chirp coefficient is accounted for as an additional input parameter. We find that the direct problem NN performs impressively in reproducing the output pulse shapes. The values of the input parameters obtained from the inverse problem network algorithm are in good agreement with the known values from the simulation data, but the estimation errors are higher than those made in the case of initially transform-limited pulses.

\section{Conclusion}

We have successfully used a supervised machine-learning model based on a NN to solve both the direct and inverse problems relating to the nonlinear pulse shaping in optical fibres. Details of this work can be found in [6]. Our results show that a properly trained network can greatly help the design and analysis of fibre-based shaping systems by providing immediate and sufficiently accurate solutions. Although demonstrated here in a fibre optics context, the principle of using NN architectures to solve wave equation-based inverse problems is expected to apply to many physical systems.

\section{References}

1. L. Michaeli and A. Bahabad, "Genetic algorithm driven spectral shaping of supercontinuum radiation in a photonic crystal fiber," J. Opt. 20, 055501 (2018).

2. S. Boscolo et al., "Performance analysis of dual-pump nonlinear amplifying loop mirror mode-locked all-fibre laser," Laser Phys. Lett. 16, 065105 (2019).

3. M. Närhi et al., "Machine learning analysis of extreme events in optical fibre modulation instability," Nat. Commun. 9, 4923 (2018).

4. C. Finot et al., "Nonlinear sculpturing of optical pulses with normally dispersive fiber-based devices," Opt. Fiber Technol. 45, 306 (2018).

5. S. Boscolo and C. Finot, Shaping Light in Nonlinear Optical Fibers (John Wiley \& Sons, Chichester, 2017).

6. S. Boscolo and C. Finot, "Artificial neural networks for nonlinear pulse shaping in optical fibers," arXiv:2002.08815 (2020). 